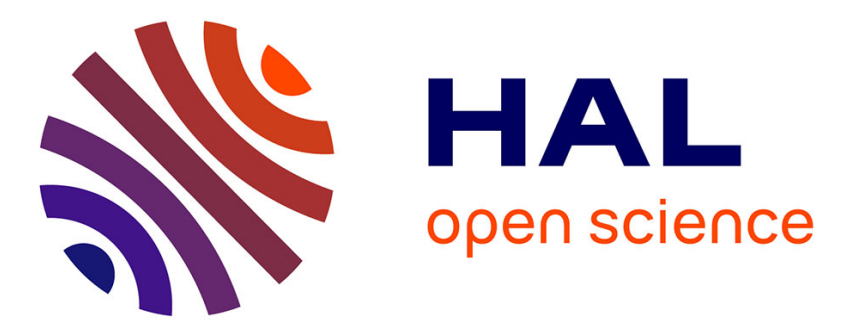

\title{
First comparison of GEANT4 hadrontherapy physics model with experimental data for a NUMEN project reaction case
}

\author{
J.R.B. Oliveira, M. Moralles, D. Flechas, D. Carbone, M. Cavallaro, D. \\ Torresi, L. Acosta, C. Agodi, P. Amador-Valenzuela, D. Bonanno, et al.
}

\section{To cite this version:}

J.R.B. Oliveira, M. Moralles, D. Flechas, D. Carbone, M. Cavallaro, et al.. First comparison of GEANT4 hadrontherapy physics model with experimental data for a NUMEN project reaction case. Eur.Phys.J.A, 2020, 56 (5), pp.153. 10.1140/epja/s10050-020-00152-6 . hal-02870812

\section{HAL Id: hal-02870812 https://hal.science/hal-02870812}

Submitted on 11 Oct 2021

HAL is a multi-disciplinary open access archive for the deposit and dissemination of scientific research documents, whether they are published or not. The documents may come from teaching and research institutions in France or abroad, or from public or private research centers.
L'archive ouverte pluridisciplinaire HAL, est destinée au dépôt et à la diffusion de documents scientifiques de niveau recherche, publiés ou non, émanant des établissements d'enseignement et de recherche français ou étrangers, des laboratoires publics ou privés. 


\title{
First comparison of GEANT4 hadrontherapy physics model with experimental data for a NUMEN project reaction case
}

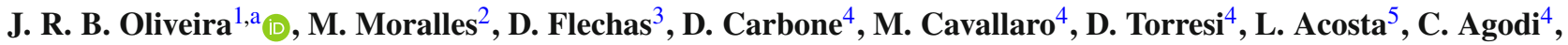 \\ P. Amador-Valenzuela ${ }^{6}$, D. Bonanno ${ }^{4}$, T. Borello-Lewin ${ }^{1}$, G. A. Brischetto ${ }^{4,7}$, S. Calabrese B $^{4,7}$ D. Calvo $^{8}$, \\ V. Capirossi ${ }^{8,9}$, F. Cappuzzello ${ }^{4,7}$, E. R. Chavez-Lomelí ${ }^{5}$, I. Ciraldo ${ }^{4,7}$, F. Delaunay ${ }^{8,9,10}$, H. Djapo $^{11}$, C. Eke ${ }^{12}$,

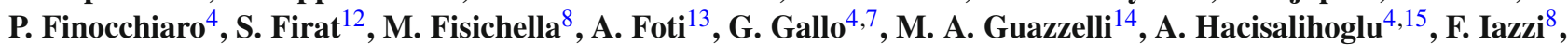 \\ R. Linares $^{16}$, D. Lo Presti ${ }^{7,13}$, J. Ma ${ }^{17}$, N. H. Medina ${ }^{1}$, A. Pakou ${ }^{18}$, L. Pandola ${ }^{4}$, H. Petrascu ${ }^{19}$, F. Pinna ${ }^{8,9}$, \\ S. Reito $^{13}$, P. Ries ${ }^{20}$, G. Russo ${ }^{7,13}$, O. Sgouros $^{4}$, S. O. Solakci ${ }^{12}$, V. Soukeras ${ }^{4}$, G. Souliotis ${ }^{21}$, A. Spatafora ${ }^{4,7}$, \\ S. Tudisco ${ }^{4}$, J. S. Wang ${ }^{17,22}$, Y. Y. Yang ${ }^{17}$, A. Yildirim ${ }^{12}$, V. A. B. Zagatto $^{16}$, for the NUMEN collaboration \\ ${ }^{1}$ Instituto de Física da Universidade de São Paulo, São Paulo, SP, Brazil \\ 2 Instituto de Pesquisas Energéticas e Nucleares, IPEN/CNEN, São Paulo, SP, Brazil \\ ${ }^{3}$ Universidad Nacional de Colombia, Bogotá, Colombia \\ ${ }^{4}$ Istituto Nazionale di Fisica Nucleare, Laboratori Nazionali del Sud (LNS), Catania, Italy \\ ${ }^{5}$ Instituto de Física, Universidad Nacional Autónoma de México, Mexico City, Mexico \\ ${ }^{6}$ Departamento de Aceleradores, Instituto Nacional de Investigaciones Nucleares, Mexico City, Mexico \\ ${ }^{7}$ Dipartimento di Fisica e Astronomia, Università di Catania, Catania, Italy \\ ${ }^{8}$ Istituto Nazionale di Fisica Nucleare, Sezione di Torino, Turin, Italy \\ ${ }^{9}$ DISAT, Politecnico di Torino, Turin, Italy \\ ${ }^{10}$ LPC Caen, Normandie Université, ENSICAEN, UNICAEN, CNRS/IN2P3, Caen, France \\ ${ }^{11}$ Institute of Accelerator Technologies, Ankara University of Physics, Ankara, Turkey \\ 12 Department of Physics, Akdeniz University, Antalya, Turkey \\ 13 Istituto Nazionale di Fisica Nucleare, Sezione di Catania, Catania, Italy \\ ${ }^{14}$ Centro Universitário FEI, São Bernardo do Campo, SP, Brazil \\ 15 Institute of Natural Science, Karadeniz Teknik Universitesi Trabzon, Trabzon, Turkey \\ ${ }^{16}$ Instituto de Física da Universidade Federal Fluminense, Niterói, RJ, Brazil \\ ${ }^{17}$ Institute of Modern Physics, Chinese Academy of Sciences, Lanzhou, China \\ ${ }^{18}$ Department of Physics and HINP, The University of Ioannina, Ioannina, Greece \\ 19 IFIN-HH, Bucarest, Romania \\ 20 Technische Universität Darmstadt, Institut für Kernphysik (IKP), Darmstadt, Germany \\ ${ }^{21}$ Laboratory of Physical Chemistry, Department of Chemistry, National and Kapodistrian University of Athens, Athens, Greece \\ 22 School of Science, Huzhou University, Huzhou, China
}

Received: 24 February 2020 / Accepted: 24 April 2020 / Published online: 26 May 2020

(c) Società Italiana di Fisica and Springer-Verlag GmbH Germany, part of Springer Nature 2020

Communicated by Jose Benlliure

\begin{abstract}
Gamma-ray and neutron spectra from the ${ }^{18} \mathrm{O}+{ }^{76} \mathrm{Se}$ reaction at $15.3 \mathrm{MeV} / \mathrm{u}$ were measured with the EDEN array of liquid scintillators at the LNS. The results were compared to GEANT Hadrontherapy physics list simulations in order to assess the reliability of this model for the development of the NUMEN project. A good agreement with the shape of the experimental gamma-ray spectra and a reasonable agreement with the total count rates were obtained. The gamma spectra originated from the nuclear reactions were selected by time coincidence with the Superconducting Cyclotron radio-frequency reference signal. The random coincidence background rate was appropriately described
\end{abstract}

only when the Faraday Cup, the material and geometry of the experimental hall and its contents were included in the simulation with sufficient detail. The information on the radiation spectra is important for the adequate development of the project of the detector arrays and electronic equipment for the advanced phase of NUMEN. Since orders of magnitude larger beam intensities are planned for this phase, the random coincidence rate is also of significant importance, particularly for the performance of the G-NUMEN gamma calorimeter array.

\footnotetext{
a e-mail: zero@if.usp.br (corresponding author)
} 


\section{Introduction}

The NUMEN project [1] is an international collaboration involving more than 30 institutions led by the Istituto Nazionale di Fisica Nucleare - Laboratori Nazionali del Sud (INFN-LNS), Catania, Italy. In this project, double charge exchange (DCE) reaction cross sections of interest to neutrino physics, particularly neutrino-less double-beta decay [2], are to be experimentally evaluated. Many heavy-ion systems have been explored in the initial phases of the NUMEN scientific program, including the system of the present study. In the advanced phase of NUMEN, very large intensity beams from the CS superconducting cyclotron accelerator (LNS) will be available for experiments [3], reaching the order of $10^{12}$ beam particles/s or more, and requiring modifications of the experimental apparatus [4-10]. Reliable estimates of the radiation field expected during such experiments are important for evaluation of the performance and possible radiation damage of detectors, electronics and other equipment. In association with the MAGNEX spectrometer [11], which will identify the species of the DCE projectile-like fragment (PLF), a gamma-array spectrometer [10] will assist the identification of the specific nuclear state which has been populated, by coincidence/anti-coincidence with the gamma transitions of the PLF and/or the target-like fragment (TLF), in the cases which MAGNEX energy resolution is insufficient for this task. With the high beam intensity, a large random coincidence rate is expected which could contaminate the DCE data. This contamination would come from uncorrelated nuclear reactions of all types of exit channels which can originate from the same beam/target combination of the entrance channel. Extensive Monte-Carlo simulations were performed for various reactions of interest to NUMEN in an attempt to provide a set of expected scenarios in which the detection system and accessories will operate, helping to choose the best design of the project. In the present work, one of these reactions $\left({ }^{18} \mathrm{O}+{ }^{76} \mathrm{Se}\right.$ at $\left.275 \mathrm{MeV}\right)$ was experimentally investigated in a first evaluation of the performance of those simulations in the project context. The EDEN array of liquid scintillators [12-14] was used for the detection of neutrons and gamma rays. The data were taken during the period of a present phase DCE measurement (November/December 2018).

Section 2 presents the description of the experimental setup and calibration procedures, Sect. 3 the simulations, Sect. 4 the results and discussion, while conclusions and perspectives are presented in Sect. 5 .

\section{The experimental setup and calibrations}

The beam, ${ }^{18} \mathrm{O}$ at $275 \mathrm{MeV}$, was provided by the CS superconducting cyclotron of the LNS, pulsed at a $20 \mathrm{MHz}$ fre- quency. About $1.5 \mathrm{nA}$ (electric) of $8^{+}$state beam ions impinged on the target, which consisted of enriched ${ }^{76} \mathrm{Se}$ $\left(274 \mu \mathrm{g} / \mathrm{cm}^{2}\right.$ thickness) on a natural C backing $\left(78 \mu \mathrm{g} / \mathrm{cm}^{2}\right.$ thickness). The target was placed at the center of the MAGNEX scattering chamber, towards which the EDEN array of detectors are oriented. The EDEN array [12-14] consists of 36 NE-213 liquid scintillator detectors capable of $n / \gamma$ discrimination. However, in the experiment only 21 detectors, those which presented the best performance in terms of energy and time resolution, noise, etc., were used in the analysis. Fast and Slow components of the pulse were separately digitized as described in [13]. The master gate for data acquisition was provided by a logical "or" of the time pulses of all detectors. The master gate in logical "and" with the RF pulse of the CS served as the common start of the time-todigital converter (TDC) with individual delayed stop signals from each detector. A data acquisition live time of $25.4 \%$ was determined for this measurement. During the experiment, the MAGNEX spectrometer was on and tuned to select the DCE PLF $\left({ }^{18} \mathrm{Ne}\right)$, since a standard NUMEN project measurement was under way. The ${ }^{18} \mathrm{O}$ beam was transported and stopped in a Faraday cup (FC) at the MAGNEX focal plane [15]. The EDEN data reported in the present work, however, were taken totally independently of the MAGNEX focal plane detector data.

The gamma-ray energy calibration of each EDEN detector was obtained in separate measurements of a ${ }^{22} \mathrm{Na}$ calibration source placed in front of each detector. In order to adjust the parameters of a linear calibration function, the spectra were compared to a GEANT4 simulation of the corresponding experimental conditions (see Sect. 3 for details). The simulation was smeared by convolution with a energy dependent width (according to Ref. [16]) Gaussian function in order to reproduce the experimental energy resolution, and normalized to show the same number of counts as the measured data between a selected threshold (around $0.25 \mathrm{MeV}$ ) and $1.5 \mathrm{MeV}$. This calibration procedure is necessary because of the low probability of photoelectric effect within the scintillator volume. Only the structure around the Compton edges of the $511 \mathrm{keV}$ and $1275 \mathrm{keV}$ gamma rays can be identified in the spectra. Figure 1a, b present typical samples of the simulation results in comparison to experimental data after calibration and subtraction of the ambient background (BG) spectra. The BG spectra were measured in a separate run with no gamma source. The correspondence is quite good except in the energy region below $0.3 \mathrm{MeV}$, where the experimental data show a relative enhancement of the number of counts probably due to the limited detail in the material surrounding the scintillator considered in the simulation. The concrete ceiling of the experimental hall, for example, which could back-scatter gamma rays towards the detectors, was not included in this simulation. 
Figure 2 shows the sum of the energy-calibrated Fast versus Slow component spectrum of all EDEN detectors obtained during the in-beam measurement. A clear separation can be observed between $\gamma$-related and neutron-related signals. Figure 3 shows the corresponding sum of the TDC timecalibrated spectra of all 21 EDEN detectors (corresponding to the dead-time corrected number of $52.5 \times 10^{10}$ beam particles hitting the target). The time range is limited within the $50 \mathrm{~ns}$ interval between beam pulses from the cyclotron. This time interval is too short for appropriate neutron time-of-flight (TOF) measurements, which were therefore not attempted. The edges of the limits are not sharp due to small variations in cable length and CFD threshold among the different detectors. The gamma-gated individual spectra of each detector present a first peak which was shifted in the time calibration to the origin of the axis ( $t=0 \mathrm{~ns})$ for simplicity. This peak is associated with the prompt gamma rays emitted from nuclear reactions with the target. A secondary peak, located at $t \approx 25 \mathrm{~ns}$ is associated with gamma transitions generated at the FC (nearly $6 \mathrm{~m}$ downstream). The intensity of this peak varies considerably among the detectors due to different amount of material shielding the radiation between the FC and the specific detector, being negligible in some of them (see Fig. 4). The structure beyond 32 ns is due, mostly, to instrumental effects related to the partial overlap of the logic time pulses of the detectors and the CS RF pulse in the coincidence module, and should be disregarded. The neutron gated spectra (e.g. red dotted line in Fig. 3) present a very flat distribution.

\section{Details of the simulations}

The experiment was simulated using two programs based on the GEANT4 Monte Carlo toolkit [17-19] version 10.04.

The first program has as input the beam parameters (ion and energy) and the target characteristics (materials and thicknesses). The environment is vacuum and the target is positioned in the center of a virtual sphere defined as a detector. Information on all reaction products that cross the surface of this sphere as well as those remaining on the target is recorded in an N-tuple object in a ROOT [20] file. The information consists of the particle identification through the PDG code [21], volume (sphere or target), energy, momentum vector and time. This program is used to simulate reactions that occur on the target as well as on the FC. The data saved in the files permit also the calculation of target and FC activation.

The second program simulates the transport of all emerging particles from the target and from the FC through the MAGNEX experimental hall and their eventual interaction with the EDEN detectors. The modeling of the experimental hall was based on a floor plan of the facility. For simplicity, besides the floor, the walls and the ceiling, only the volumes

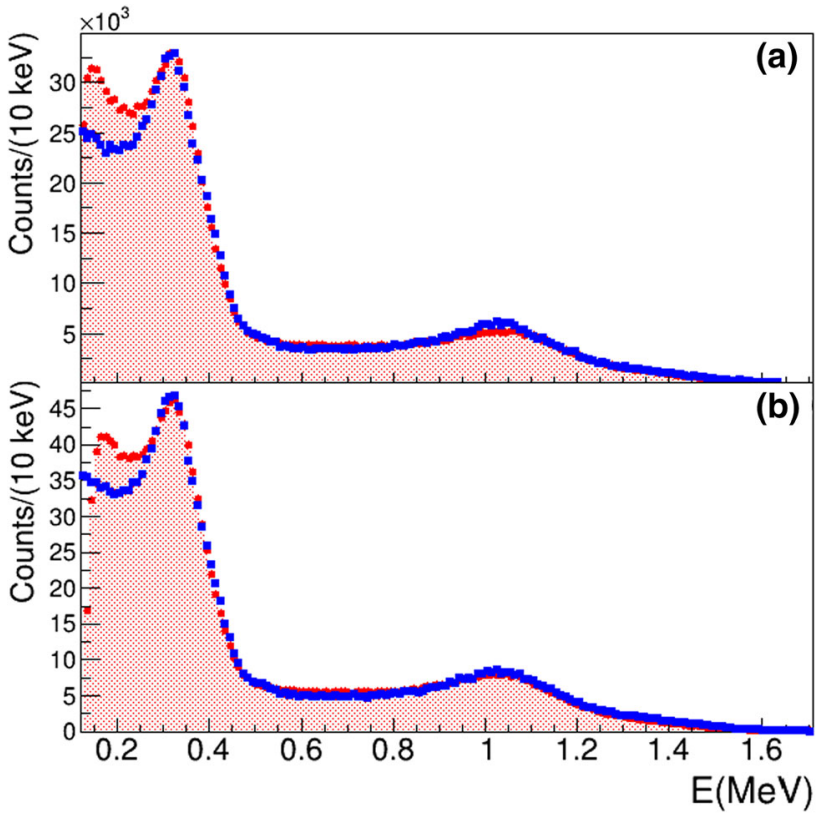

Fig. 1 Typical samples of the energy calibrated spectra in comparison with the corresponding simulation for a ${ }^{22} \mathrm{Na}$ source placed close to the face of each detector. The error bars (standard deviation) are smaller than the symbols. Hatched, in red: Experimental spectra for 2 different detectors, (a, b), after energy calibration. Blue square data points: simulation

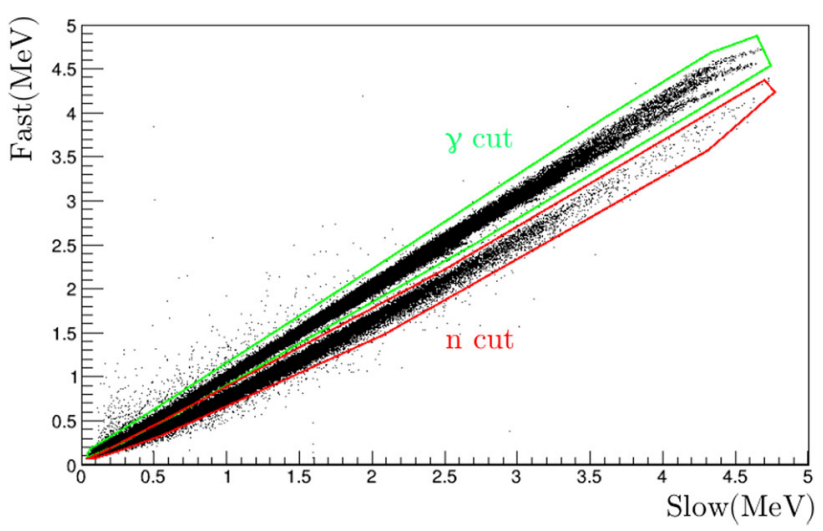

Fig. 2 Sum of all energy calibrated Fast vs Slow component 2D histograms showing the $\gamma$ (green) and $n$ (red) cuts. The scales (in MeV) are valid for the energy deposited by $\gamma$-rays by primary interaction with electrons. For neutrons, they correspond to electron-equivalent (ee) values, which are less than the actual energy deposited by the interactions (mainly with protons) in the detector

with major contributions to scattering and absorption of particles were included in the simulation. These volumes are simplified versions of the EDEN detectors, the scattering chamber, the magnetic quadrupole and dipole of the MAGNEX, the focal plane detector and the FC. Each EDEN detector was simplified by one cylindrical volume $(20 \mathrm{~cm}$ diameter by 5 $\mathrm{cm}$ length) with the scintillating material and a tube-shaped volume for its outer casing. Figure 5 shows a schematic of the 


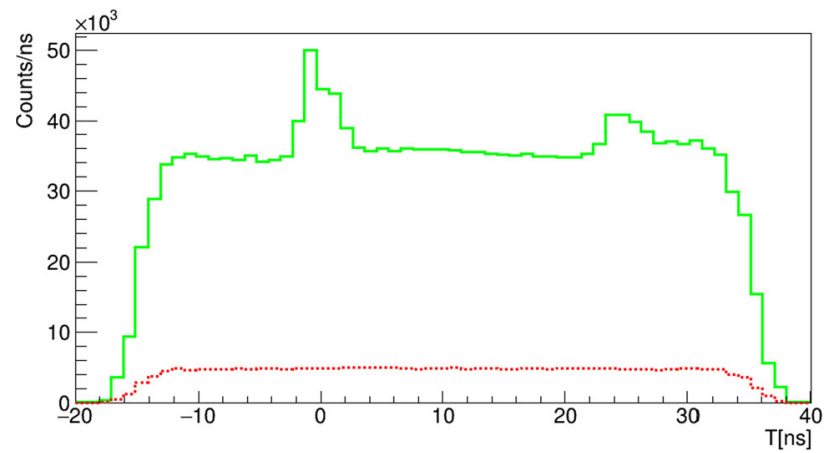

Fig. 3 Sum of all calibrated $\gamma$-selected (solid green line) and $n$ selected (dashed red line) time spectra. A requirement of one and only one detector hit with the detector deposited energy range corresponding to $0.25<E<2.5 \mathrm{MeVee}$ (electron equivalent) was imposed

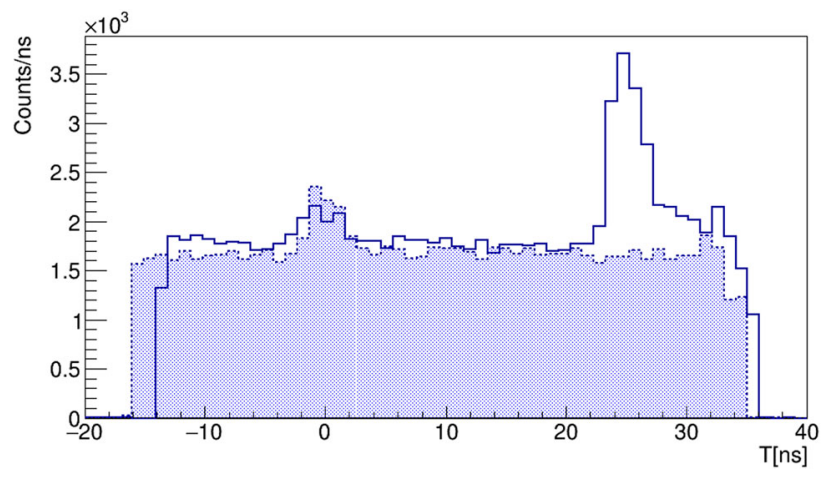

Fig. 4 Typical samples of the energy calibrated gamma time spectra of 2 different EDEN detectors (number 6, clear, and number 29, shaded spectrum)

GEANT4 modeled assembly. This program has as input the output file of the first program. It produces another file with $\mathrm{N}$-tuples containing the PDG code, the time and the energy deposited in the scintillator for all particles that interact with the EDEN detectors. The recording and reading of the files was done using GEANT4's specific library to handle ROOT files. Note that in the case of the interaction with neutrons (mainly with protons) the energy deposited into the detector active volume is not converted to the detection pulse electric charge with the same proportionality constant as that of electrons (e.g. from gamma-ray interactions). No attempt to convert the deposited energy to the integrated pulse charge was attempted in the simulations. Therefore, care should be taken when comparing the electron-equivalent values, which are the ones experimentally obtained for neutrons, with the simulations.

The sensitive material of the EDEN detectors is the NE213 liquid scintillator. The properties of this scintillator that allows gamma and neutron discrimination by pulse shape analysis have been well studied. GEANT4 has already been successfully used by Hartwig and Gumplinger [22] and by Scherzinger et al. [23] to simulate the NE-213 scintillators

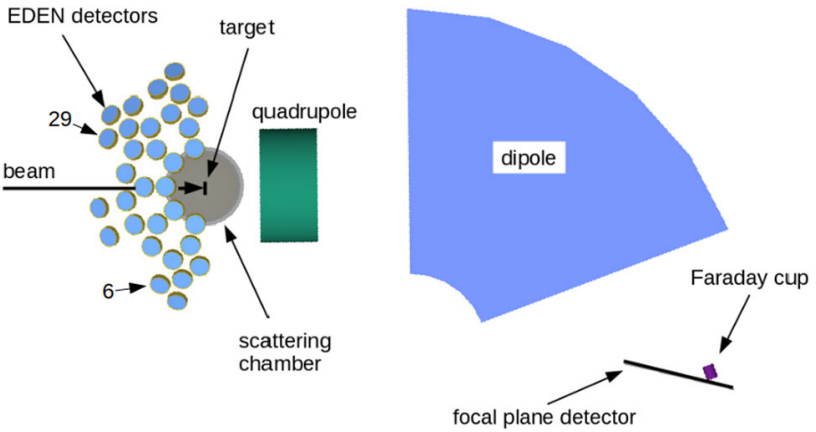

Fig. 5 The MAGNEX and EDEN setup modeled in GEANT4. The location of detectors number $6\left(\theta=105^{\circ}, \phi=126^{\circ}\right)$ and number 29 $\left(\theta=122^{\circ}, \phi=60^{\circ}\right)$ mentioned in the caption of Fig. 4 are indicated

response to gammas and neutrons. As mentioned above, we have also successfully simulated the detector response to a ${ }^{22} \mathrm{Na}$ calibration source (Fig. 1).

The typical reactions explored in the NUMEN experiments employ beams of ${ }^{18} \mathrm{O}$ and ${ }^{20} \mathrm{Ne}$ with energies ranging from 15 to $60 \mathrm{MeV} / \mathrm{u}$. The present measurement was incorporated into one of these experiments, which in the present phase are concentrated near $15 \mathrm{MeV} / \mathrm{u}$. Among the options of physical models to simulate these reactions, we chose the set of processes used in the hadrontherapy example provided by the GEANT4 distribution, namely the physics list called "Hadrontherapy1". This option includes the well established processes for electromagnetic interactions that are responsible for the transport of photons and charged particles through the materials and the ParticleHP package which includes high-precision models for neutrons and light charged particles. It contains also the physical processes which describe the decay of excited nuclei and the transport and interactions of neutrons. The "G4BinaryLightIonReaction" physics model was used for the nucleus-nucleus collisions, which is an extension of the Binary Intranuclear Cascade model (BIC) for reactions with light ions [24]. Comparisons of this and other GEANT4 physics models (QMD-Quantum Molecular Dynamics, and INCL-Liège Intranuclear Cascade) with experimental results were reported for ${ }^{12} \mathrm{C}$ beam at $95 \mathrm{MeV} / \mathrm{u}$ on different targets [25], 55.6 MeV/u on human tissues [26], $400 \mathrm{MeV} / \mathrm{u}$ on water [27], and $62 \mathrm{MeV} / \mathrm{u}$ on ${ }^{\text {nat }} \mathrm{C}$ [28]. None of these models appears to outperform the others in a general sense, while they were all tested in a higher energy range than in our case. We consider the BIC model suitable for our purpose of describing the background produced by reactions with cross sections that are several orders of magnitude larger than the DCE reactions. 


\section{Results and discussion}

\subsection{Gamma-ray spectra}

Figure 6 shows the sum of the gamma cut energy spectra determined from the Slow pulse component (similar results were also obtained using the Fast component) of all detectors, gated in time around the prompt peak $(-2.5 \mathrm{~ns}<t<4.5 \mathrm{~ns})$ and accidental-coincidence subtracted, in comparison to the corresponding simulation, following $14 \times 10^{10}$ primaries, normalized to the same number of projectiles of the experiment $\left(N_{p}=45.0(14) \times 10^{10}\right.$, determined from the integrated FC charge, dead-time corrected). The shape of the simulation spectrum fits well the experimental one above $E>0.25$ $\mathrm{MeV}$ (i.e. above threshold of all detectors). The integral of the simulation spectrum in the interval between $E=0.25$ and $2.5 \mathrm{MeV}$ (within the active range of all detectors) corresponds to the experimental one if multiplied by a factor of 0.80. Although this indicates an overestimation of a factor of 1.26(4) by the simulation, the result is quite remarkable, considering the complexity and variety of nuclear reactions that can be involved in such a measurement.

\subsection{Gamma-ray fold distribution}

Table 1 presents the experimental coincidence gamma-ray fold $K$ (number of EDEN detectors fired within the -2.5 to $4.5 \mathrm{~ns}$ time gate) distribution in comparison to the simulated one. The simulation results were multiplied by the number of projectiles normalization factor (3.2) in order to correspond to the experimental value of $N_{p}=45 \times 10^{10}$. An accidental coincidence $\mathrm{BG}$ time gate was set in the range from -11.5 to $-4.5 \mathrm{~ns}$ for the experimental data. The accidental BG was subtracted from the data assuming a flat uncorrelated time distribution from -11.5 to 4.5 ns (i.e. comprising the peak range) and a binomial combination of the two time gates, the fold $K$ being defined when all $K$ events occur within the time peak gate. The uncertainties presented in the table are from counting statistics origin only. An additional systematic error of about $4 \%$ can be estimated from the normalization factor and other origins like the choice of specific energy and time gate widths. For $K=2$ the simulation underestimates the experimental rate by a factor of about 2 , thus in opposite direction to the $K=1$ trend. This means that the average gamma multiplicity of the nuclear reactions is larger than expected by the simulation, and/or the Compton scattering between detectors is more frequent in the experiment. This last hypothesis is less probable since the actual amount of structure material between detectors is about the same or somewhat larger than modelled for the simulation, which should lead to a small reduction of the Compton cross talk due to absorption. For $K=3$ the experimental result is dominated by the random coincidence statistics (absent in

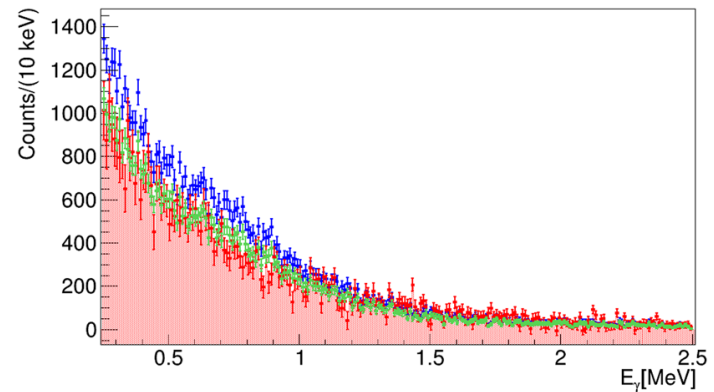

Fig. 6 Sum of the gamma spectra of all detectors, gated on the (target associated) time peak and $K=1$, accidental BG subtracted (red, shaded). The corresponding simulation is shown with blue data points. The same simulation spectrum multiplied by a factor of 0.80 is shown in green. The error bar in this and in all other figures represent 1 statistical standard deviation

the simulation) and only an upper limit could be evaluated. The value is apparently smaller than the simulation value but consistent with it within two error bars.

\subsection{Gamma-ray angular distribution}

Figure 7 presents the angular distribution of the ratio $R_{S / E}$ between the simulated and the experimental integrals for $K=1$ (in the same energy interval: $0.25-1.5 \mathrm{MeV}$ ) of the prompt gamma spectrum of each detector as a function of its (a) polar angle $\left(\theta_{L A B}\right)$ and (b) azimuthal angle $\left(\phi_{L A B}\right.$, with reference on the horizontal plane, pointing to the left, looking downstream). The values of the ratio lie between 1.0 and 1.5 (the dashed lines in Fig. 7), with exception of the two data points above 2.0 [in both (a) and (b) plots] of largest azimuthal angles, for which there may be larger absorption by interposed material between the target and the detectors in the experiment than considered in the simulation. Since the geometry of the equipment material in this region is rather complex, no attempt was made to improve the simulation. With exclusion of these two data points [in both (a) and (b)] one can say that there is a rather constant $R_{S / E}$ ratio as a function of either angle. The data points distribution then shows a mean value of 1.22 with a standard deviation of 0.15 . The rather small variations might be due to imperfect modelling of the target chamber material geometry in the simulation. Figure 8 presents both the experimental and simulated gamma detection angular distributions. Again excluding the abnormal data points (in this plot, those below 1500 counts), it appears that there is no strong dependence of the count rate with polar angle.

\subsection{Gamma-ray and neutron time spectra}

As it is apparent in Fig. 3, a considerable amount of (flat) accidental coincidence BG is present in the experimental time spectrum. In order to obtain the corresponding simu- 
Table 1 Gamma-ray fold distribution data. Number of cases corresponding to $N_{p}=45 \times 10^{10}$ (the FC integrated value, dead-time corrected) ${ }^{18} \mathrm{O}$ projectiles impinging on the target

\begin{tabular}{llll}
\hline$K$ & Experiment & Simulation & S/E ratio \\
\hline 1 & $4.95(7) \times 10^{4}$ & $6.25(4) \times 10^{4}$ & $1.26(2)$ \\
2 & $2.04(5) \times 10^{3}$ & $0.84(5) \times 10^{3}$ & $0.41(3)$ \\
3 & $<8$ & $16(7)$ & $>0.5$ \\
\hline
\end{tabular}
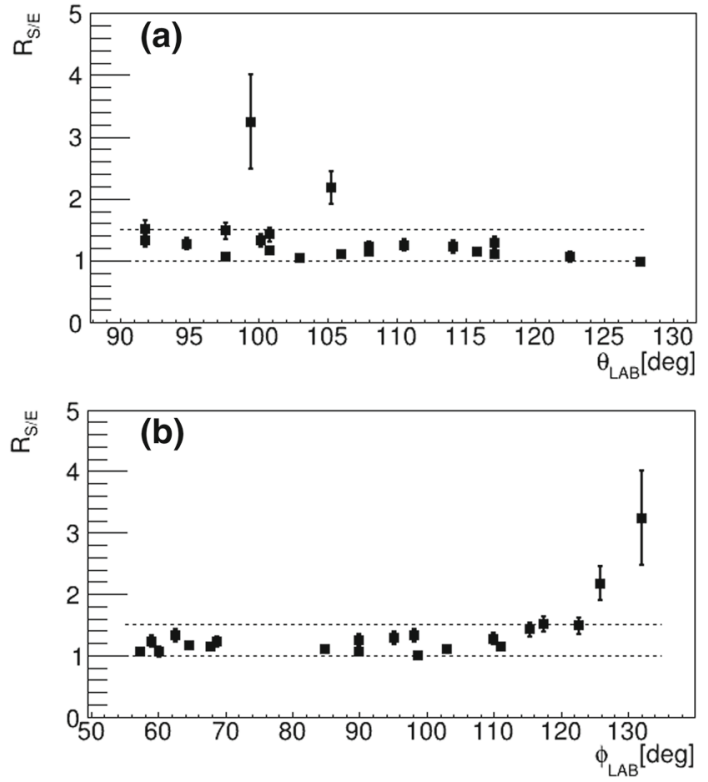

Fig. 7 The ratio $R_{S / E}$ between the simulated and the experimental integrals of the prompt gamma spectrum of each EDEN detector as a function of its laboratory frame a polar angle $\left(\theta_{L A B}\right)$ and $\mathbf{b}$ azimuthal angle $\left(\phi_{L A B}\right)$

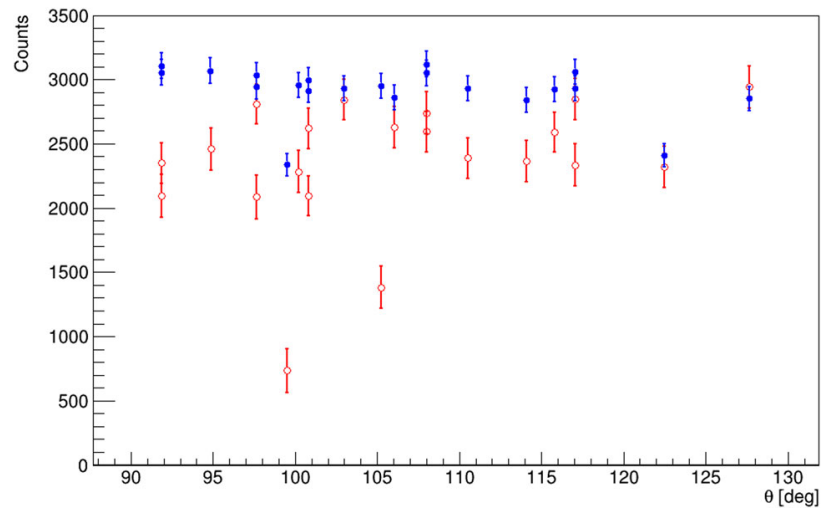

Fig. 8 The angular distribution of simulated (blue closed circles) and experimental (red open circles) detection of gamma rays in laboratory frame polar angle lated spectrum, the modulo operation has to be applied to the time value of the simulated data, reproducing the effect of the cyclotron beam period which limits the range of the experimental time spectrum to $T_{b}=50 \mathrm{~ns}$. Figure 9 presents the simulated time spectrum of the target originated events, where the time values were obtained from the original ones $\left(T_{0}\right)$ according to Eq. 1:

$T=\left(T_{0}+t_{p}\right) \bmod T_{b}-t_{r}$

The parameters $t_{p}=9 \mathrm{~ns}$ and $t_{r}=15 \mathrm{~ns}$ were adjusted to reproduce the peak position and range of the experimental spectrum, respectively. However, the spectrum of Fig. 9 shows a very small accidental BG (for example, the region below $T \approx-4 \mathrm{~ns}$ ). To the right of the prompt peak a broader structure of delayed events appears with a maximum around $T=9 \mathrm{~ns}$, decaying back to the height of the accidental event rate towards the end of the time range at $35 \mathrm{~ns}$. The presence of this structure is not clear in Fig. 3, possibly due to the larger background of the experimental data. The experimental gamma spectrum shows also the secondary time peak at around $T=25 \mathrm{~ns}$ produced by the interaction of the beam with the FC. This time value is consistent with the sum of the delay of the ${ }^{18} \mathrm{O}$ beam ions to reach the $\mathrm{FC}$ (about $111 \mathrm{~ns}$ along the $6 \mathrm{~m}$ ion optical trajectory distance), with the delay of the gamma rays form the FC back to the Eden detectors (around $20 \mathrm{~ns}$ ), minus the delay of the gamma rays from the target to the Eden detectors ( $6 \mathrm{~ns}$ ), minus 2 beam periods (100 $\mathrm{ns})$. Separate simulations were then performed including the interaction of the beam with the FC (consisting of aluminium sufficiently thick to stop the beam). The simulations were performed with and without the material of the concrete walls (floor and ceiling) of the MAGNEX experimental hall. The results are presented in Fig. 10. In this case a value of $t_{p}=19$ ns was used in Eq. 1 in order to reproduce the location of the experimental peak, due to the different time reference of the simulations for the FC events (which do not include the beam time-of flight). It is clear from these results that the presence of the walls is paramount for the increase of the accidental BG proportion in the spectra. The gamma BG increases by a factor of 36, while neutron one increases by a factor of 6 . This can be attributed mostly to scattering of neutrons and gamma-ray production by secondary neutron reactions from the walls. It is to be noted that the concrete walls were already 


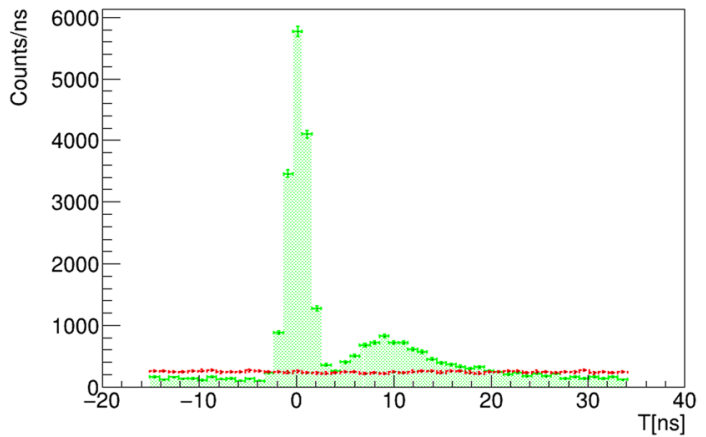

Fig. 9 Simulated time spectra from target originated events. Green, shaded: gamma rays. Red: neutrons. The deposited energies were limited to the $0.25-2.5 \mathrm{MeVee}$ range

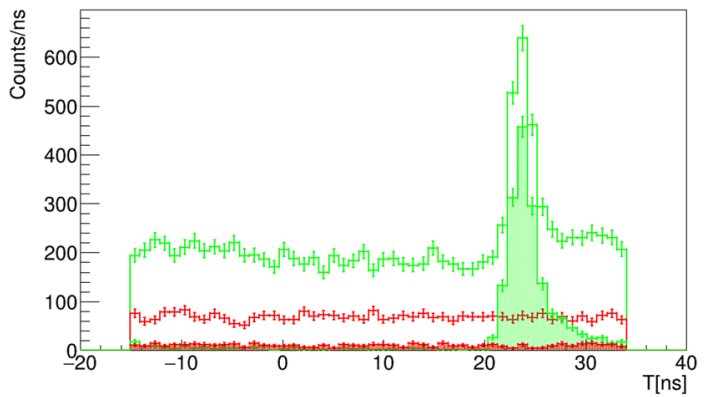

Fig. 10 Simulated time spectra from FC originated events. Green: gamma rays (peaking near $24 \mathrm{~ns}$ ). Red: neutrons. The shaded spectra were obtained without the presence of the experimental hall walls. The deposited energies were limited to the $0.25-2.5 \mathrm{MeVee}$ range

included in the target simulation of Fig. 9, with a very small increase in the background. This means that the main source of neutrons which contribute to the accidental BG is, by far, the beam interactions with the FC.

Figure 11 presents the time sum spectra of the target and FC originated events of the simulation in comparison to the experimental results, for gammas and neutrons. The simulation has a good correspondence with the experiment for gamma rays. The target prompt and accidental BG levels are similar. The secondary FC prompt peak, however, is overestimated by the simulation. The magnitude of this peak is compatible with zero, within error bars, for both the simulation and the experiment for all detectors below $\phi_{L A B}=100^{\circ}$. Above this value, corresponding to the detectors which are un-shielded or only partially shielded by the MAGNEX iron (the bottom seven EDEN detectors in Fig. 5), this peak is significant in both experiment and simulation, but the simulation overestimates the experiment by one order of magnitude or more, depending on the detector. This is, in part, due to discrepancy between the actual material interposed between the FC and some of the detectors of the EDEN array, but it is also possible that it comes from deviations of the actual production of gamma radiation in the $\mathrm{FC}$ with respect to the simulation. Indeed it is not expected that the reaction model

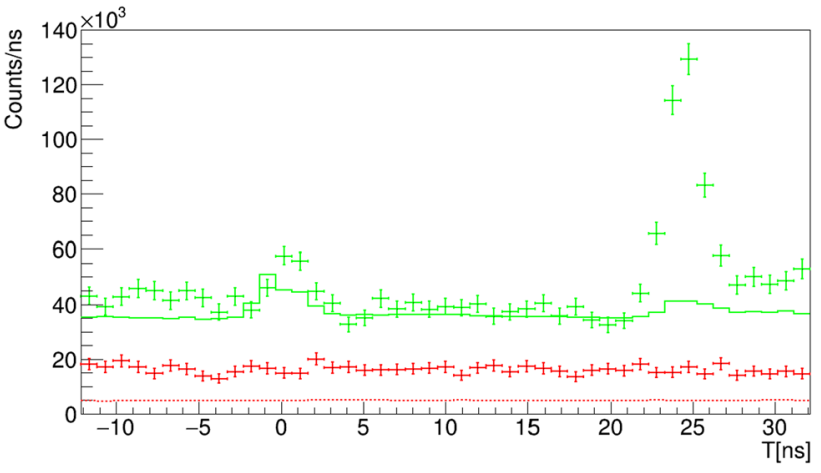

Fig. 11 Simulated time sum spectra from target and FC originated events in comparison to the experimental ones. Experimental results: Green solid line for gamma rays and red dashed line for neutrons. Green and red data points with error bars are the simulations for gammas and neutrons, respectively. The deposited energies were limited to the 0.25 2.5 MeVee range

would remain good down to very low beam energies. Further measurement are necessary for a reliable evaluation of this issue. The figure also indicates a larger rate of neutrons in the simulation in comparison to the experiment, but this can be an artifact in the simulation due to the different neutron deposited energy threshold of $1 \mathrm{MeV}$ assumed to correspond to $0.25 \mathrm{MeVee}$ electron-equivalent deposit from the neutron interaction with protons in the detector, and less importantly, the correspondence of $5 \mathrm{MeV}$ to the upper level discrimination of $2.5 \mathrm{MeVee}$. These values are a rough estimate from [23] in which a Fast/Slow decomposition of the pulse was performed in similarity to our case. Unfortunately we do not have experimental values specifically for our case, since the $50 \mathrm{~ns}$ range was insufficient for neutron time of flight (TOF) measurements. Again more detailed experiments including TOF are in order for a deeper investigation.

Figure 12 presents the (FC origin) accidental BG simulation (normalized by the number of beam particles) in comparison to the experimental results for gamma rays, as a function of the azimuthal angle $\left(\phi_{L A B}\right)$ of each EDEN detector. It can be seen that a slowly rising distribution is observed, in mutual compatibility. The rise of the simulation points above $110^{\circ}$ might be due to the smaller than real absorption of the FC related events in the simulation for this angular region as already pointed out. The diffuseness of the origin of this secondary radiation should explain the relatively small variation with angle, in contrast to the secondary time peak, which is detected directly from the FC.

\section{Conclusions and perspectives}

The prompt gamma spectrum of the ${ }^{18} \mathrm{O}+{ }^{76}$ Se reaction at $275 \mathrm{MeV}$ was measured with the NE-213 scintillators of the EDEN array. The shape of the energy spectrum was well 


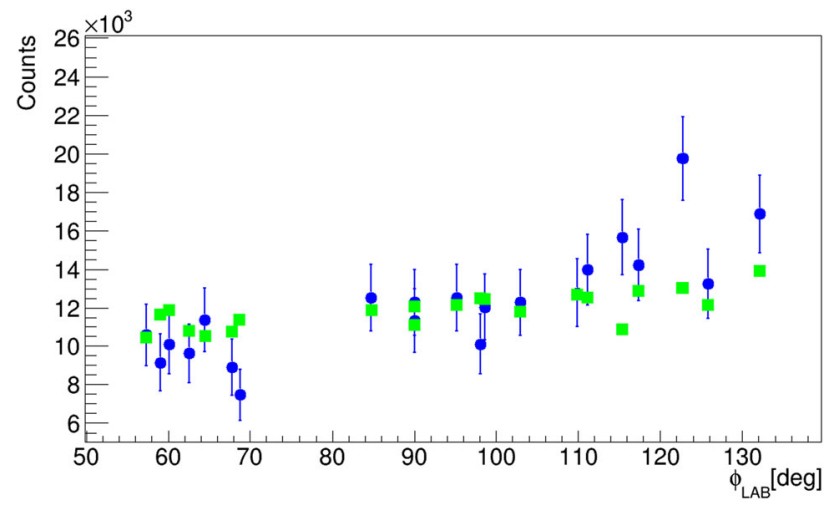

Fig. 12 Number of gamma-ray accidental BG events (corresponding to $45 \times 10^{10}$ beam particles) in the -11.5 to $-4.5 \mathrm{~ns}$ time range as a function of the azimuthal angle (laboratory frame). Simulation (FC origin only): Blue closed circles with error bars. Experiment: Green solid squares (error bars smaller than symbols). The deposited energies were limited to the $0.25-2.5 \mathrm{MeV}$ range

reproduced by GEANT4 simulations with the "Hadrontherapy 1" physics list. The integral of the spectrum in the 0.25 $2.5 \mathrm{MeV}$ was reasonably well reproduced, although overestimated by $26(4) \%$, which can be considered a quite good result in face of the complexity of the task. A similar result, obtained in a different context (proton therapy monitoring, with protons at somewhat higher energy per nucleon) was reported in Ref. [29].

The angular distribution of the gamma rays was also well described in the $\theta=92^{\circ}-128^{\circ}$ angular range of the detector array. Two-fold $(K=2)$ gamma coincidences (gamma signals within the $7 \mathrm{~ns}$ width gate around the prompt peak) were observed with about $4 \%$ of the intensity of the $K=1$ rate, in the same energy range, and were underestimated by the simulation, indicating a possible underestimation of the average multiplicity of gamma rays produced by the nuclear reactions, and/or, less likely, an underestimation of the Compton cross talk between detectors.

The order of magnitude of accidental gamma BG (the flat portion of the time spectrum) was also well reproduced by the simulation after the inclusion of the events originated by the reactions of the beam with the Faraday cup a few meters away downstream from the target, at the MAGNEX focal plane, and the secondary scattering and nuclear reactions of the FC generated neutrons (mainly) with the MAGNEX dipole and quadrupole iron cores and the concrete walls of the experimental hall.

The neutron spectra were also measured with the EDEN detectors, and, differently from the gamma rays, presented a very flat distribution in the $50 \mathrm{~ns}$ range between cyclotron accelerator beam pulses. This flat distribution was appropriately described by the simulations after restraining the neutron event deposited energies to the $1-5 \mathrm{MeV}$ range, which was roughly estimated to correspond to the $0.25-2.5 \mathrm{MeVee}$ accepted range of the detector array electronics. The order of magnitude of the count rate appears to be well reproduced, but this result is uncertain due to the lack of a precise knowledge of the neutron detection energy calibration of the system used.

The results obtained in the present work are important for the NUMEN project, and encourage the use of the GEANT4 hadrontherapy model for the production of reliable predictions supporting the design of the future detection system. Care should be taken, however, to not overstate and generalize the good results of this specific case to all reactions and energies to be used in the NUMEN project. Additional measurements of other reactions and energies should be performed for a more comprehensive validation of the simulations, including neutron time-of-flight measurements, if possible. The importance of the inclusion of the material of the experimental hall and heavy equipment has also been evidenced by the present results. The accidental coincidence BG is a very relevant issue particularly for the design and performance prediction of the future gamma calorimeter array of the NUMEN project. It has become apparent that the bunker shielding of the beam dump which will have to be designed has to be very effective in reducing the radiation emanating from it and bringing the secondary reactions with the material of the experimental hall to an acceptable level.

The validation of nuclear reaction models in MonteCarlo simulations is also important for medical physics (e.g. hadrontherapy) $[25-27,29,30]$ and other applications such as high intensity beam accelerators for secondary radioactive beam production, and accelerator driven reactors. Experimental data for this purpose is still rather scarce in the literature.

Acknowledgements We acknowledge support from Fundação de Amparo à pesquisa no Estado de São Paulo, (FAPESP grants proc. 2016/04612-9 and 2017/50160-5), Conselho Nacional de Desenvolvimento Científico e Tecnológico, CNPq and from Instituto Nacional de Ciência e Tecnologia-Física Nuclear e Aplicações (INCT-FNA, research project 464898/2014-5), Brazil.

Data Availability Statement This manuscript has no associated data or the data will not be deposited. [Authors' comment: Data can be made available uppon request, together with explanations of their acquisition conditions.]".

\section{References}

1. F. Cappuzzello et al., Eur. Phys. J. A 54, 72 (2018)

2. H. Lenske et al., Prog. Part. Nucl. Phys. 109, 103716 (2019)

3. L. Calabretta et al., Mod. Phys. Lett. A 32, 17 (2017)

4. F. Iazzi et al., WIT Trans. Eng. Sci. 116, 61 (2017)

5. F. Pinna, D. Calvo, V. Capirossi, F. Delaunay, M. Fisichella, F. Iazzi, R. Introzzi, Il Nuovo Cimento 42C, 67 (2019)

6. A. Muoio et al., Eur. Phys. J. Web Conf. 117, 10006 (2016)

7. S. Tudisco et al., Sensors 18(7), 2289 (2018) 
8. C. Ciampi, et al., NIMA Vol. 925 (2019) Nuclear Instum. Meth. A 925, 60 (2019)

9. D. Carbone et al., Results Phys. 6, 863 (2016)

10. J.R.B. Oliveira et al., J. Phys. Conf. Ser. 1056, 012040 (2018)

11. F. Cappuzzello et al., Eur. Phys. J. A 52, 167 (2016)

12. H. Laurent, H. Lefort, D. Beaumel, Y. Blumenfeld, S. Fortier, S. Galès, J. Guillot, J.C. Roynette, P. Volkov, Nucl. Instrum. Meth. A 326, 517 (1993)

13. M. Cavallaro et al., Nucl. Instrum. Meth. A 700, 65 (2013)

14. M. Cavallaro et al., Phys. Rev. C 93, 064323 (2016)

15. M. Cavallaro et al., Nucl. Instrum. Methods B 463, 334 (2020)

16. M. Cecconello, M. Donato, C. Marini-Bettolo, S. Conroy, S. Sangaroon, G. Ericsson, Nucl. Instrum. Methods A 753, 34 (2014)

17. S. Agostinelli et al., Nucl. Instrum. Methods A 506, 250 (2003)

18. J. Allison et al., IEEE Trans. Nucl. Sci. 53, 270 (2006)

19. J. Allison et al., Nucl. Instrum. Methods A 835, 186 (2016)

20. R. Brun, F. Rademakers, Nucl. Instrum. Methods A 389, 81 (1997)

21. M. Tanabashi et al., Particle Data Group. Phys. Rev. D 98, 030001 (2018)

22. Zachary S. Hartwig, Peter Gumplinger, Nucl. Instrum. Methods A 737, 155 (2014)
23. J. Scherzinger, R. Al Jebali, J.R.M. Annand, K.G. Fissum, R. HallWilton, K. Kanaki, M. Lundin, B. Nilsson, H. Perrey, A. Rosborg, H. Svensson, Nucl. Instrum. Methods A 840, 121 (2016)

24. G. Folger, V.N. Ivanchenko, J.P. Wellisch, Eur. Phys. J. A 21, 407 (2004)

25. J. Dudouet, D. Cussol, D. Durand, M. Labalme, Phys. Rev. C 89 , 054616 (2014)

26. M. De Napoli, F. Romano, D. D’Urso, T. Licciardello, C. Agodi, G. Candiano, F. Cappuzzello, G.A.P. Cirrone, G. Cuttone, A. Musumarra, L. Pandola, V. Scuderi, Phys. Med. Biol. 59, 7643 (2014)

27. D. Bolst, G.A.P. Cirrone, G. Cuttone, G. Folger, S. Incerti, V. Ivanchenko, T. Koi, D. Mancusi, L. Pandola, F. Romano, A.B. Rosenfeld, S. Guatelli, Nucl. Instrum. Methods A 869, 68 (2017)

28. C. Mancini-Terracciano et al., World Congress on Medical Physics and Biomedical Engineering 2018, IFMBE Proceedings 68, 675

29. M. Pinto, D. Dauvergne, N. Freud, J. Krimmer, J.M. Létang, E. Testa, Front. Oncol. 6, 10 (2016)

30. C. Mancini-Terracciano et al., Phys. Med. 67, 116 (2019) 
European Physical Journal A -- Hadrons \& Nuclei is a copyright of Springer, 2020. All Rights Reserved. 